1 Rapid and simultaneous screening of 47,XXY and $A Z F$ microdeletions by quadruplex

\title{
real-time polymerase chain reaction
}

\section{Abstract}

We developed a quadruplex real-time PCR assay that allows rapid and simultaneous detection of 47,XXY and azoospermia factor $(A Z F)$ microdeletions on $\mathrm{Y}$ chromosome. The quadruplex assay consisted of four hydrolysis probes and primer sets. Three probes and the corresponding primers were used to qualitatively detect $A Z F \mathrm{a}, A Z F \mathrm{~b}$, and $A Z F \mathrm{c}$ deletions. For the detection of 47,XXY, the hydrolysis probe-mediated melting analysis was conducted to analyze the relative amounts of $\mathrm{X}$ and $\mathrm{Y}$ chromosomes. The quadruplex assay for detecting 47,XXY was characterized by very high analytical specificity $(100 \%)$ in a wide template DNA range (2-100 ng). The detection limit of the assay was $2 \mathrm{ng}$ of genomic DNA, and the optimal template DNA amount for the detection of 47,XXY was $25 \mathrm{ng}$. The quadruplex assay for detecting 47,XXY and $A Z F$ microdeletions has also demonstrated very high diagnostic sensitivity and specificity (100\%). The assay was found to be rapid, sensitive, reliable, and inexpensive. This method is suggested to be applied as a first-step tool in genetic screening of patients with non-obstructive azoospermia and severe oligospermia.

Key words: $A Z F$ microdeletion, azoospermia, 47,XXY, oligospermia, real-time PCR

\section{Introduction}

Genetic abnormalities, including cytogenetic and molecular abnormalities, account for $15 \%-30 \%$ of male infertility by affecting spermatogenesis and sperm transport [1]. The 
identification of certain genetic abnormalities not only helps to clarify the causes of infertility but also helps to guide treatment options (e.g., assisted reproduction) and evaluate prognosis. Nowadays, it is well recognized that men with non-obstructive azoospermia and severe oligospermia (NOASO) should undergo karyotyping and Y-chromosomal microdeletions (YCMD) tests for the detection of cytogenetic and molecular abnormalities before assisted reproduction [2].

$47, \mathrm{XXY}$ is the most frequent genetic cause of NOASO and accounts for approximately two-thirds of all cytogenetic abnormalities observed in infertile men [3]. The diagnosis of 47,XXY provides the patients with an explanation about their life experiences and possible mental and physical therapies, thus providing major benefits to their quality of life [4]. YCMD is the second most frequent genetic cause of spermatogenetic failure in NOASO after 47,XXY [5]. Most clinically relevant YCMD occur in azoospermia factor $(A Z F)$ regions located on the long arm of the $\mathrm{Y}$ chromosome, which is classically subdivided into $A Z F \mathrm{a}$, $A Z F \mathrm{~b}$, and $A Z F \mathrm{c}$ regions. The specific location of the deletion influences its impact on the therapeutic options and prognosis [6]. 47,XXY and AZF microdeletions are mostly identified using karyotyping and multiplex PCR, respectively [7]. However, the increasing demand of genetic tests may pose a challenge for methods characterized by a limited throughput. Furthermore, current tests for the detection of deletions are highly sensitive to carry-over contaminations that frequently occur during post-PCR manipulations (e.g., electrophoresis). In the previous study, we have developed a quadruplex real-time PCR assay for detection of $A Z F$ microdeletions [8]. The assay was validated to be fast, contamination-free, easy to setup and automated. In the current study, we modified the previous assay to endow it with an 
additional capability to detect 47,XXY. By measuring the analytical and diagnostic sensitivities and specificities of the modified assay, we evaluated its effectiveness as a first-step genetic screening tool for men with NOASO.

\section{Materials and methods}

\subsection{Principle of quadruplex assay}

The quadruplex assay consisted of four hydrolysis probes and primer sets to qualitatively detect $A Z F$ microdeletions and 47,XXY. Specifically, HEX-, ROX-, and Cy5-labeled probes and their corresponding primers were designed to detect the target sequence tagged sites (STSs) located on $A Z F \mathrm{a}, A Z F \mathrm{~b}$, and $A Z F \mathrm{c}$ regions of the $\mathrm{Y}$ chromosome, respectively. The deletion of a specific $A Z F$ region will result in the absence of the amplification curve in the corresponding fluorescent channel. Therefore, real-time amplification enabled us to obtain information concerning $A Z F$ microdeletion from the HEX, ROX, and CY5 channels (Fig. 1A).

Melting analysis along with amplification of segmental duplications was performed to detect 47,XXY. Specifically, segmental duplications, which are DNA segments of nearly identical sequences, are widespread in the human genome [9]. Specific segmental duplications located on chromosomes $\mathrm{X}$ and $\mathrm{Y}$ can be amplified simultaneously with a single primer set to generate two amplification products, while preserving the original ratio of the two sequences [10]. Based on this principle, a FAM-labeled probe and its corresponding primers were designed to amplify and detect segmental duplicate sequences located on the $\mathrm{X}$ and $\mathrm{Y}$ chromosomes. To protect the FAM-labeled probe from hydrolysis during amplification, the 
melting temperature of such probe was designed to be lower than the annealing temperature of PCR. Therefore, the amplification curve could not be observed in the FAM channel during the amplification (Fig. 1A). After amplification, PCR products were subjected to melting analysis. As shown in Fig. 1B, during the melting process, FAM-labeled probe was hybridized with two paralogous target sequences. The target sequence on the $\mathrm{X}$ chromosome completely matched the probe, while that of Y chromosome only partially matched, generating two distinct peaks in the resulting melting curve. Theoretically, the ratio of the two peak areas indicates the relative amounts of paralogous PCR products, which indirectly indicates the relative amounts of $\mathrm{X}$ and $\mathrm{Y}$ chromosomes. To reduce the variance between tests and equipments, we used 46,XY samples as reference, and introduced the normalized relative peak (NRP) value for the detection of 47,XXY (Fig. 1C). Following this theory, NRP values of a 46,XY sample and a 47,XXY sample would be 1 and 0.5 , respectively.

\subsection{Quadruplex real-time polymerase chain reaction}

Quadruplex real-time PCR amplification and melting analysis were performed on a LightCycler 480 II thermocycler (Roche Applied Science GmbH, Mannheim, Germany). We designed two quadruplex reactions to evaluate the method. According to the STSs and segmental duplications recommended by the literature $[7-8,10]$, reaction A consisted of $\mathrm{X} / \mathrm{Y}-1$, sY86, sY127, and sY254 primers and probes; and reaction B consisted of X/Y-2, sY84, sY134, and sY255 primers and probes. The $25-\mu \mathrm{L}$ reaction mixtures contained $10 \mathrm{mmol} / \mathrm{L}$ Tris- $\mathrm{HCl}$ (pH 8.3), $50 \mathrm{mmol} / \mathrm{L} \mathrm{KCl,} 1 \mathrm{U}$ TaqHS (Takara Bio, Dalian, China), $3.0 \mathrm{mmol} / \mathrm{L} \mathrm{Mg}^{2+}$, and 0.2 $\mathrm{mmol} / \mathrm{L}$ of each deoxynucleoside triphosphate. To optimize the quadruplex reaction, we 
introduced the homo-tag assisted non-dimer system in which all the primers had a common sequence at their $5^{\prime}$ ends to generate an universal primer binding region, and such sequence was also used as the universal primer [11]. Detailed primers and probes information are summarized in Table. 1 . The cycling conditions were: $95^{\circ} \mathrm{C}$ for $3 \mathrm{~min}$, followed by 40 cycles of $95^{\circ} \mathrm{C}$ for $15 \mathrm{~s}, 63^{\circ} \mathrm{C}$ for $20 \mathrm{~s}$, and $72^{\circ} \mathrm{C}$ for $20 \mathrm{~s}$. Fluorescence data from the four corresponding channels were collected at the end of the annealing step. The melting analysis began with denaturation at $95^{\circ} \mathrm{C}$ for $1 \mathrm{~min}$ and re-naturation at $25^{\circ} \mathrm{C}$ for $1 \mathrm{~min}$, followed by melting from $25^{\circ} \mathrm{C}$ to $70^{\circ} \mathrm{C}$ with a ramp rate of $0.14^{\circ} \mathrm{C} / \mathrm{s}$. Fluorescence signals were collected at each degree centigrade.

\subsection{Analytical sensitivity and specificity of quadruplex assay}

Analytical sensitivity refers to the minimum number of copies in a sample that can be measured accurately with the assay, and analytical specificity refers to the PCR assay detecting the appropriate target sequence rather than other, nonspecific targets [12].To detect 47,XXY, first we evaluated the analytical specificity and sensitivity of the quadruplex assays by differentiating the 46,XY and 47,XXY samples. We collected peripheral blood-originated DNA samples of 47,XXY patients and 46,XY men $(n=12$ /karyotype, the sample size has been approved by statistical justification, please see supplemental materials) from the molecular diagnostic laboratory of Xiamen Maternal and Child Health Hospital (Xiamen, China). To evaluate the analytical specificity, 46,XY and 47,XXY samples were differentiated using reaction A and reaction B. To evaluate the analytical sensitivity, the specificity study was conducted with $100,50,25,12.5,5,2$, or 1 ng of DNA template. Four random 46,XY 
samples were used as reference samples to calculate the NRP value.

To detect $A Z F$ microdeletions, we also evaluated the analytical specificity of the quadruplex assays by differentiating five samples bearing different $A Z F$ microdeletions ( $A Z F \mathrm{a}$, $A Z F \mathrm{~b}, A Z F \mathrm{c}, A Z F \mathrm{bc}$, and $A Z F \mathrm{abc}$ ) and three DNA control samples (unaffected male, female, and no-template control).

\subsection{Diagnostic sensitivity and specificity of quadruplex assay}

Diagnostic sensitivity is the percentage of individuals with a given condition whom the assay identifies as positive for that condition, and diagnostic specificity is the percentage of individuals without a given condition whom the assay identifies as negative for that condition [12]. To evaluate the diagnostic sensitivity and specificity of the quadruplex assay, ninety blind peripheral blood-originated DNA samples (25 ng), including 46,XY without AZF microdeletions ( $\mathrm{n}=30), 47, \mathrm{XXY}(\mathrm{n}=30)$ and $A Z F$ microdeletions ( $\mathrm{n}=30)$, were tested under reaction A and reaction B conditions. For the 47, XXY detection, the reference intervals were calculated with 12 known samples used in the analytical sensitivity study, and the NRP values were calculated with the identical reference samples used in the analytical sensitivity study.

For reaction $\mathrm{A}$ and reaction $\mathrm{B}$, reference intervals were defined as mean $\pm 2 \mathrm{SD}$ and mean \pm 3 SD to cover $95 \%$ and $99 \%$ confidence intervals, respectively.

\subsection{Reference methods and DNA extraction}

The reference methods for the detection of 47,XXY and $A Z F$ microdeletions were karyotyping and EAA/EMQN guideline method [7], respectively. For karyotyping, 30 
cultured leucocytes were analyzed for each 47,XXY karyotype, and 20 cultured leucocytes were analyzed for each 46,XY karyotype. In these samples, there was no evidence of mosaicism. Genomic DNA was extracted from $200 \mu \mathrm{L}$ of blood samples using the QIAamp DNA Blood mini Kit (QIAGEN GmbH, Hilden, Germany) according to the manufacturer's protocol. The concentration of extracted genomic DNA was determined by measuring the UV absorbance at $260 \mathrm{~nm}$ with the NanoVue plus spectrophotometer (GE Healthcare

Bio-Sciences Corp, NJ, USA). The DNA samples used in this study were obtained from the remainders of previous karyotyping or YCMD tests. Since information other than results of karyotype and YCMD (e.g., names and ages of the patients) was strictly withheld from the study group, no written consent was required. The study protocol was approved by the Research Ethics Committee of Xiamen Maternal and Child Health Hospital.

\subsection{Statistical analysis}

Analytical specificity of quadruplex assay for the $47, \mathrm{XXY}$ detection was estimated by the one-way analysis of variance (ANOVA) (please see supplemental materials for detail) [13]. Statistical analyses were performed with OriginPro 7.5 software (OriginLab Corp, MA, USA).

\section{Results}

\subsection{Analytical sensitivity and specificity of quadruplex assay}

The two karyotypes, 47, XXY and 46, XY, were distinctly differentiated from each other at all template levels (1-100 ng; Fig. 2A) and there was no overlap of NRP values between the 
karyotypes when 2-100 ng of DNA was used as template (Fig. 2B). Therefore, the quadruplex assay for detecting 47,XXY was applicable in the wide template amount range studied and the detection limit was $2 \mathrm{ng}$ of genomic DNA. In addition, $25 \mathrm{ng}$ of DNA was found to be the optimal template amount for detection of 47,XXY (Fig. 2C).

Detection of $A Z F$ microdeletions was characterized by the same sensitivity range (2-100 ng DNA) as that found for 47,XXY detection (Fig. 3). Samples containing all types of $A Z F$ microdeletions and unaffected male control samples were explicitly differentiated. Moreover, no false-positive signals were observed for female and no-template controls (Fig. 4).

Consequently, the detection of $A Z F$ microdeletions by means of the quadruplex assay demonstrate an analytical specificity of $100 \%$.

\subsection{Diagnostic sensitivity and specificity of quadruplex assay}

Results from the quadruplex assay were consistent with those obtained with the reference methods (Tab. 2). The remaining 60 samples were then estimated for the detection of 47,XXY. As shown in Fig. 5, for reaction A, 28 samples were classified as 46,XY and 28 samples were classified as 47,XXY based on the reference intervals; for reaction B, 29 samples were classified as 46,XY and 30 samples were classified as 47,XXY. ("first test" in fig. 5)

Indistinguishable samples in the first tests were re-tested and successfully classified ("second test" in fig. 5). The subsequent verification also revealed the complete consistency between the quadruplex assay and reference method (Tab. 2). Therefore, in a blind test with 90 samples, the quadruplex assay attained $100 \%$ diagnostic sensitivity and specificity for the detection of 47,XXY and $A Z F$ microdeletions. 


\section{Discussion}

Compared to the multiplex PCR assay currently used for basic YCMD detection [7], the assay described in the present paper does not increase the cost for each sample, because the increased cost incurred by using the hydrolysis probe is neutralized by the cost decrease due to the elimination of electrophoresis. Furthermore, our method reduces labor intensity and turnaround time (from overnight to $1.5 \mathrm{~h}$ ) and increases test throughput. The most important advances associated with our method are its ability to detect 47,XXY and $A Z F$ microdeletions simultaneously, and avoiding the carry-over contamination associated with electrophoresis that carries a considerable risk of false negatives in deletion detection. 
abnormalities such as 47,XXY detection than for the detection of deletions such as YCMD.

It is known that approximately $10 \%$ of $47, \mathrm{XXY}$ is presented as mosaicism (e.g., 47,XXY/46,XY) [16]. Theoretically, NRP values of mosaicism are between 0.5 and 1. In practice, samples whose NRP values are distributed between reference intervals of 47,XXY and $46, \mathrm{XY}$ should be further confirmed by other methods such as karyotyping, to measure the mosaic status. Apart from 47,XXY and AZF microdeletions, the other less frequent genetic causes of spermatogenetic failure such as XX male syndrome and 47,XYY syndrome, could be theoretically detected by the quadruplex assay [17]. In terms of the XX male syndrome, the lack of the $A Z F$ region would result in the absence of the amplification curve in the HEX, ROX, and CY5 channels; furthermore, the lack of the segmental duplicate sequence on the Y chromosome would generate one-peak melting profile. In terms of 47,XYY, the theoretical NRP value should be 2. However, further data from the corresponding samples should be collected to validate the effectiveness of the quadruplex assay for the detection of these two syndromes.

It should be noted that the reference samples that were used for NRP calculation were 46,XY male without $A Z F$ microdeletions, so the amplification within a reaction was quadruplex. In contrast, for samples bearing $A Z F$ microdeletions, the amplification within a reaction was less than quadruplicate. Because of the amplification imbalance between reference and $A Z F$ deletion samples, the NRP algorithm for the detection of 47,XXY was incompatible with the samples bearing $A Z F$ microdeletions. Therefore, we differentiated $A Z F$ microdeletions first, and the deletion-positive samples were not examined with the NRP algorithm for the detection of $47, \mathrm{XXY}$. Such procedures may pose a finite risk for missing the 
detection of an extra $\mathrm{X}$ chromosome in patients simultaneously affected by $47, \mathrm{XXY}$ and $A Z F$ microdeletions. However, since there is no convincing evidence supporting the finding that clinically relevant $A Z F$ microdeletions occur in patients with 47,XXY, the clinical consequences are likely to be insignificant [18-19].

In conclusion, here, we provided technical evidence that our rapid, inexpensive quadruplex real-time PCR assay is reliable for simultaneous detection of 47,XXY and AZF microdeletions. This method could be applied as a first-step genetic screening of patients with NOASO in fertility clinics after multicenter validation with a large sample size. Since other known or unknown chromosomal aberrations could also be associated with NOASO, samples with negative results in our screening test should further be examined by karyotyping and/or other molecular methods (e.g., chromosomal microarray or high-throughput sequencing) to comprehensively determine the genetic cause of male infertility [14-15].

\section{Acknowledgements}

We would like to thank Drs Yanwei Sha and Honggen Ou'yang for sample collection, Dr. Wei Zhang for statistic analysis, and Cytogenetic Laboratory of Xiamen Maternal and Child Health Hospital for providing samples and karyotype data. This work were supported by National Science Foundation for Young Scholars of China (project no. 81201361), Foundation for Young Scholars of Fujian Provincial Department of Health Office (project no. 2010-2-111), and Fujian provincial science and technology key project (project no. 2012D066).

\section{Disclosure statement}


No competing financial interests exist.

\section{Reference}

[1] Plaseski T, Noveski P, Trivodalieva S, Efremov GD, Plaseska-Karanfilska D. Quantitative fluorescent-PCR detection of sex chromosome aneuploidies and AZF deletions/duplications.

Genet Test 2008; 12:595-605.

[2] Diagnostic evaluation of the infertile male: a committee opinion. Fertil Steril 2012; 98:294-301.

[3] De Braekeleer M, Dao TN. Cytogenetic studies in male infertility: a review. Hum Reprod $1991 ; 6: 245-250$.

[4] Lanfranco F, Kamischke A, Zitzmann M, Nieschlag E. Klinefelter's syndrome. Lancet $2004 ; 364: 273-283$.

[5] Foresta C, Moro E, Ferlin A. Y chromosome microdeletions and alterations of spermatogenesis. Endocr Rev 2001; 22:226-239.

[6] Krausz C, Quintana-Murci L, McElreavey K. Prognostic value of Y deletion analysis: what is the clinical prognostic value of Y chromosome microdeletion analysis? Hum Reprod 2000; 15:1431-1434.

260 [7] Krausz C, Hoefsloot L, Simoni M, Tuttelmann F. EAA/EMQN best practice guidelines for molecular diagnosis of Y-chromosomal microdeletions: state-of-the-art 2013. Andrology $2014 ; 2: 5-19$.

[8] Guo Q, Lan F, Xu L, Jiang Y, Xiao L, Huang H, Zhou Y. Quadruplex real-time polymerase chain reaction assay for molecular diagnosis of Y-chromosomal microdeletions. 
266 [9] Bailey JA, Yavor AM, Massa HF, Trask BJ, Eichler EE. Segmental duplications:

267 organization and impact within the current human genome project assembly. Genome Res $268 \quad 2001 ; 11: 1005-1017$.

[10]Guo Q, Xiao L, Zhou Y. Rapid diagnosis of aneuploidy by high-resolution melting

270 analysis of segmental duplications. Clin Chem 2012; 58:1019-1025.

271 [11] Brownie J, Shawcross S, Theaker J, Whitcombe D, Ferrie R, Newton C, et al. The

272 elimination of primer-dimer accumulation in PCR. Nucleic Acids Res 1997; 25:3235-3241.

273 [12]Bustin SA, Benes V, Garson JA, Hellemans J, Huggett J, Kubista M, Mueller R, Nolan T,

274 et al. The MIQE guidelines: minimum information for publication of quantitative real-time

275 PCR experiments. Clin Chem 2009; 55:611-622.

276 [13]Bewick V, Cheek L, Ball J. Statistics review 9: one-way analysis of variance. Crit Care $277 \quad 2004 ; 8: 130-136$.

278 [14]Rajcan-Separovic E. Chromosome microarrays in human reproduction. Hum Reprod Update 2012; 18:555-567.

[15]Xu M, Qin Y, Qu J, Lu C, Wang Y, Wu W, Song L, Wang S, et al. Evaluation of five candidate genes from GWAS for association with oligozoospermia in a Han Chinese population. PLoS One 2013; 8:e80374.

[16]Bojesen A, Juul S, Gravholt CH. Prenatal and postnatal prevalence of Klinefelter syndrome: a national registry study. J Clin Endocrinol Metab 2003; 88:622-626. 
287 Assist Reprod Genet 2011; 28:1091-1098.

288 [18] Simoni M, Tuttelmann F, Gromoll J, Nieschlag E. Clinical consequences of

289 microdeletions of the Y chromosome: the extended Munster experience. Reprod Biomed

290 Online 2008; 16:289-303.

291 [19]Tuttelmann F, Gromoll J. Novel genetic aspects of Klinefelter's syndrome. Mol Hum 292 Reprod 2010; 16:386-395.

293 
Figure 1. Principle of quadruplex real-time PCR assay. (A) Quadruplex real-time amplification with hydrolysis probe detection. This process takes approximately 1.2 hours. Different colors indicate different fluorescent labeled hydrolysis probes. Information on Azoospermia factor $(A Z F)$ microdeletions was obtained from the HEX (for AZFa), ROX (for $\mathrm{AZFb}$ ) and CY5 (for AZFc) channels. (B) Melting analysis of segmental duplicates for the detection of 47,XXY. This process takes approximately 0.2 hours. Specific peak profiles were generated in FAM channel by melting analysis. Theoretically, the ratio of the two peak areas indirectly indicates the relative amounts of $\mathrm{X}$ and $\mathrm{Y}$ chromosomes. Tm: the melting temperature; the temperature at which half of the hydrolysis probes are dissociated from the template DNA. (C) Normalized relative peak (NRP) algorithm for 47,XXY detection. NRP value was used in $47, \mathrm{XXY}$ detection to reduce the differences between the tests or equipments. The reference sample: male $46 \mathrm{XY}$ sample unaffected by $A Z F$ microdeletions. 
Figure 2. Analytical sensitivity and specificity of the quadruplex assay for the detection of 47,XXY. Normalized relative peak (NRP) values for two karyotypes (circles: 46,XY; triangles: 47, XXY, n=12/ karyotype) determined at 100, 50, 25, 12.5, 5, 2 or $1 \mathrm{ng}$ of template amount (A) presented as median (the line within a box), mean (the square within a box), interval

312 between the $25^{\text {th }}$ and $75^{\text {th }}$ percentiles (the horizontal borders of each box) and the interval

313 between the mean \pm 2 SD (whiskers) (B). $F$ value increases with the differences between the

314 two groups, was used as the parameter for quantitatively measuring analytical specificity (C).

315 There is no overlap of NRP values between the 47,XXY and 46,XY samples when 2-100 ng

316 of DNA was used as template. The highest analytical specificity was reached in reactions A

317 and reaction B when $25 \mathrm{ng}$ of DNA was used as a template. 
320 Figure 3. Analytical sensitivity of the quadruplex assay for the detection of $A Z F$

321 microdeletions. 100, 50, 25, 12.5, 5, and 2 ng of genomic DNA (derived from 46,XY male

322 without $A Z F$ microdeletions) were analyzed using quadruplex assay. The six template levels

323 can be robustly detected in HEX, ROX, and CY5 channels.

324

325 
Figure 4. Analytical specificity of the quadruplex assays for the detection of $A Z F$

327 microdeletions. Samples containing five types of $A Z F$ microdeletions $(A Z F \mathrm{a}, A Z F \mathrm{~b}, A Z F \mathrm{c}$,

$328 A Z F \mathrm{bc}$, and $A Z F \mathrm{abc}$ ) and unaffected male control samples were explicitly differentiated.

329 Moreover, no false-positive signals were observed for female and no-template controls.

330

331 
332 Figure 5. Diagnostic specificity of quadruplex assay for the detection of 47,XXY. Sixty

333 samples including thirty 47,XXY samples and 30 unaffected male controls were differentiated

334 by quadruplex assays. For reaction A, 28 samples were classified as $46, \mathrm{XY}$ and 28 samples

335 were classified as $47, \mathrm{XXY}$ based on the reference intervals; for reaction B, 29 samples were

336 classified as 46,XY and 30 samples were classified as 47,XXY (first test). Indistinguishable

337 samples in the first tests were re-tested and successfully classified (second test). 
A Quadruplex real-time amplification

$1.2 \mathrm{~h}$

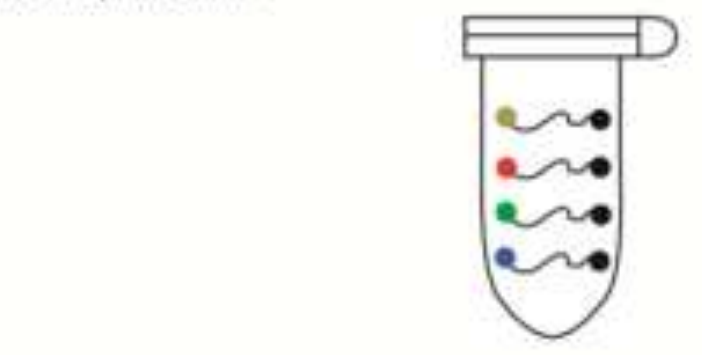

Y-chromosomal microdeletions detection

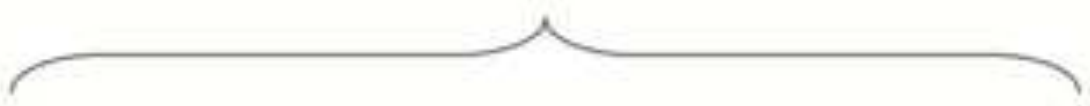

+ Unaflected

- Deletion
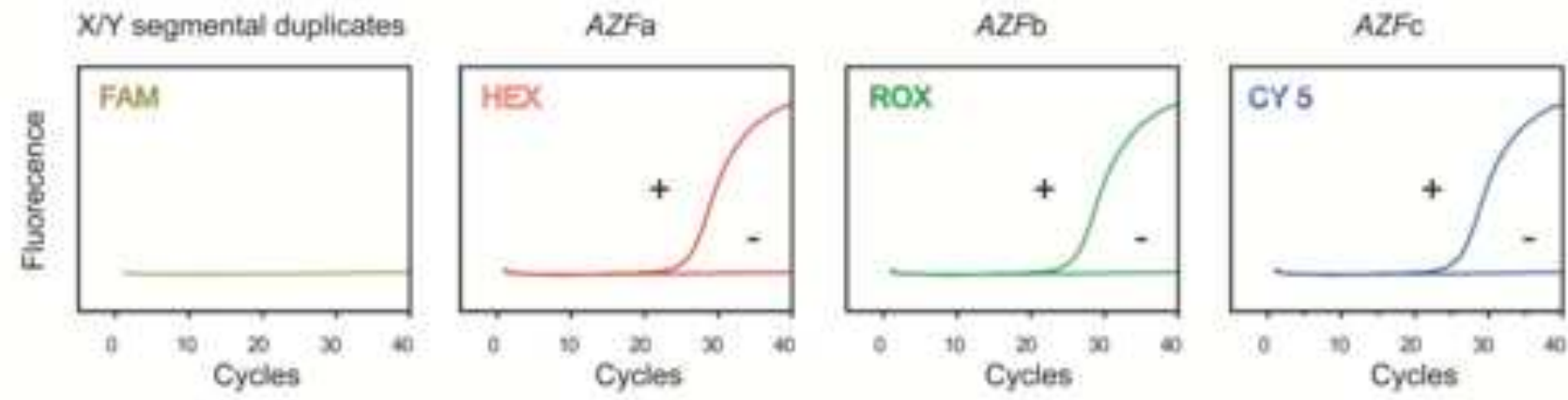

B Melting analysis

5. O-taccoctgtactgac111111111111111

3. $T m=58^{\circ} \mathrm{C}$

Chromosome $\mathrm{X}$

CTMGTGAACGGTTCTICTI - ATGGCGACATGACTG - ACCTCTCGGTGTTCGAC

$0.2 \mathrm{~h}$

5 - O-tacccotoractcac- $3^{\prime}$ Tmiatc

$1111 \mid 111111111$

Chromosome Y CTRGIGGAACGGTTCTTCTI- ALGGTGACATGACTG - ACCTCTCGGTGTTCGAC

46. $X Y$

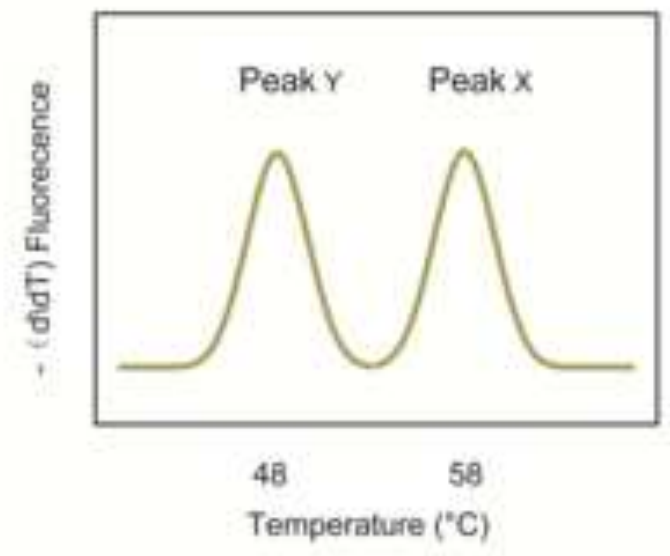

47, XXY

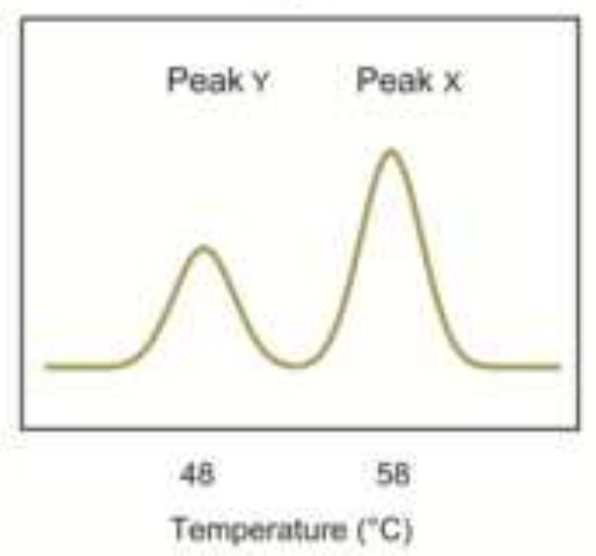

C Data analysis for $47, \mathrm{XXY}$ detection

$$
\begin{aligned}
\text { Relative peak (RP) value } & =\frac{\text { Peak } \mathrm{Y} \text { area }}{\text { Peak } \times \text { area }} \\
\text { Normalized relative peak (NRP) value } & =\frac{R P \text { value of target sample }}{\text { RP value of reference sample }}
\end{aligned}
$$


Figure 2

A

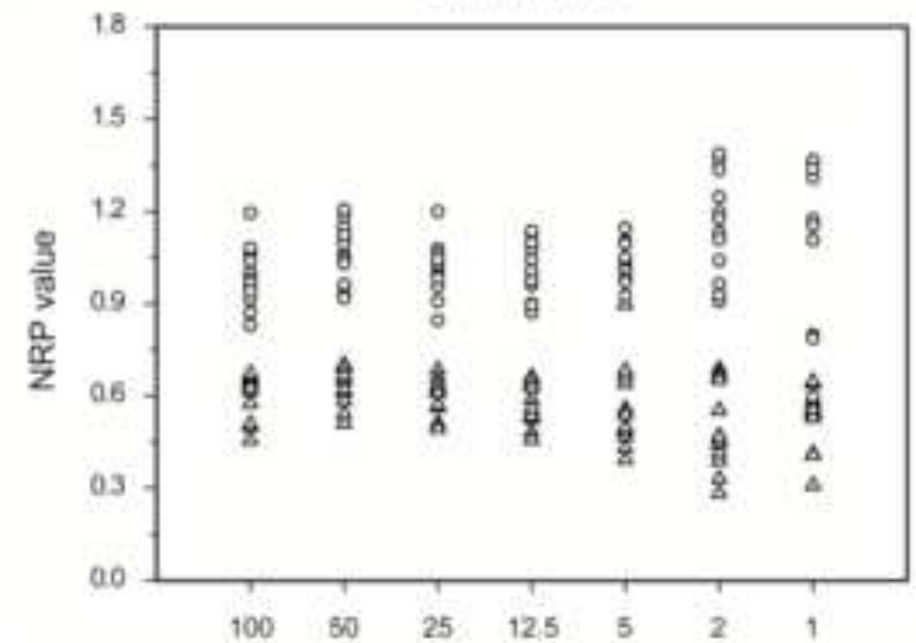

B

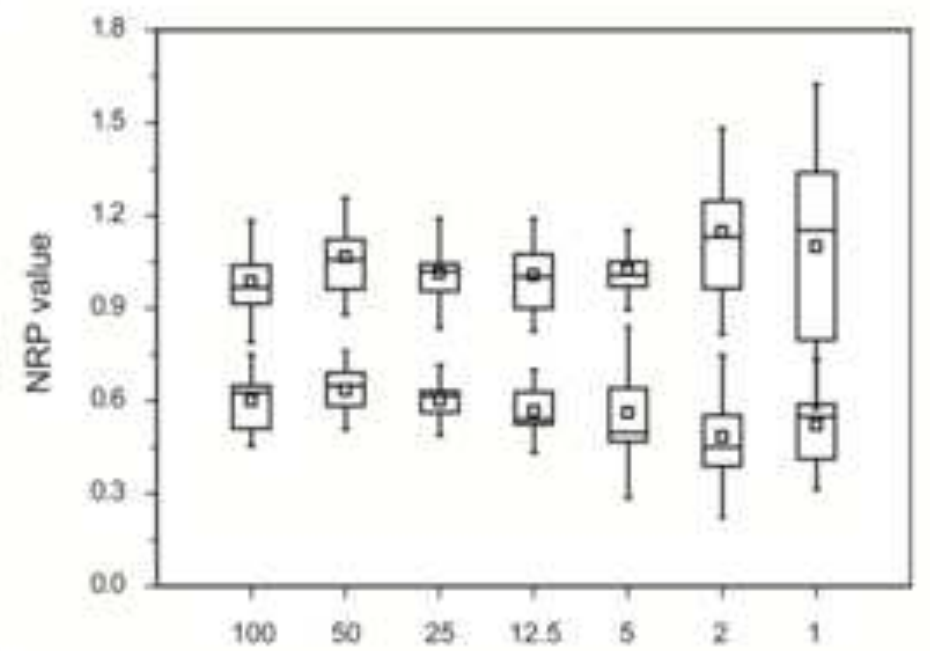

C

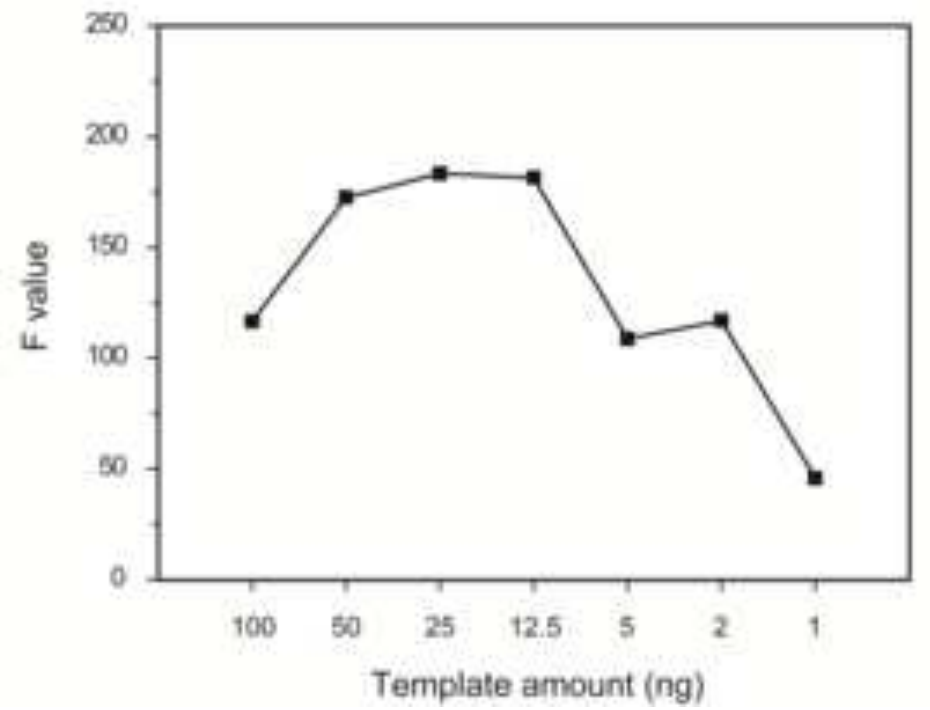

Reaction B
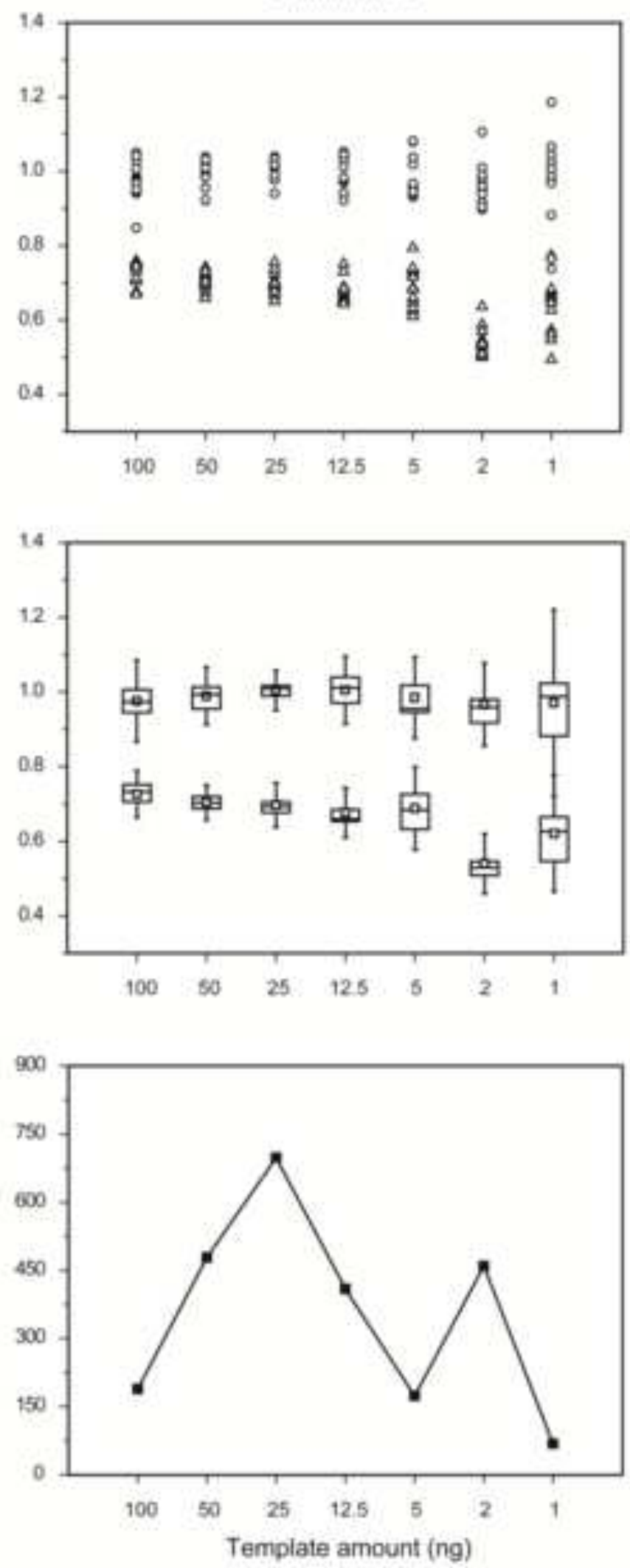


\section{Reaction A}
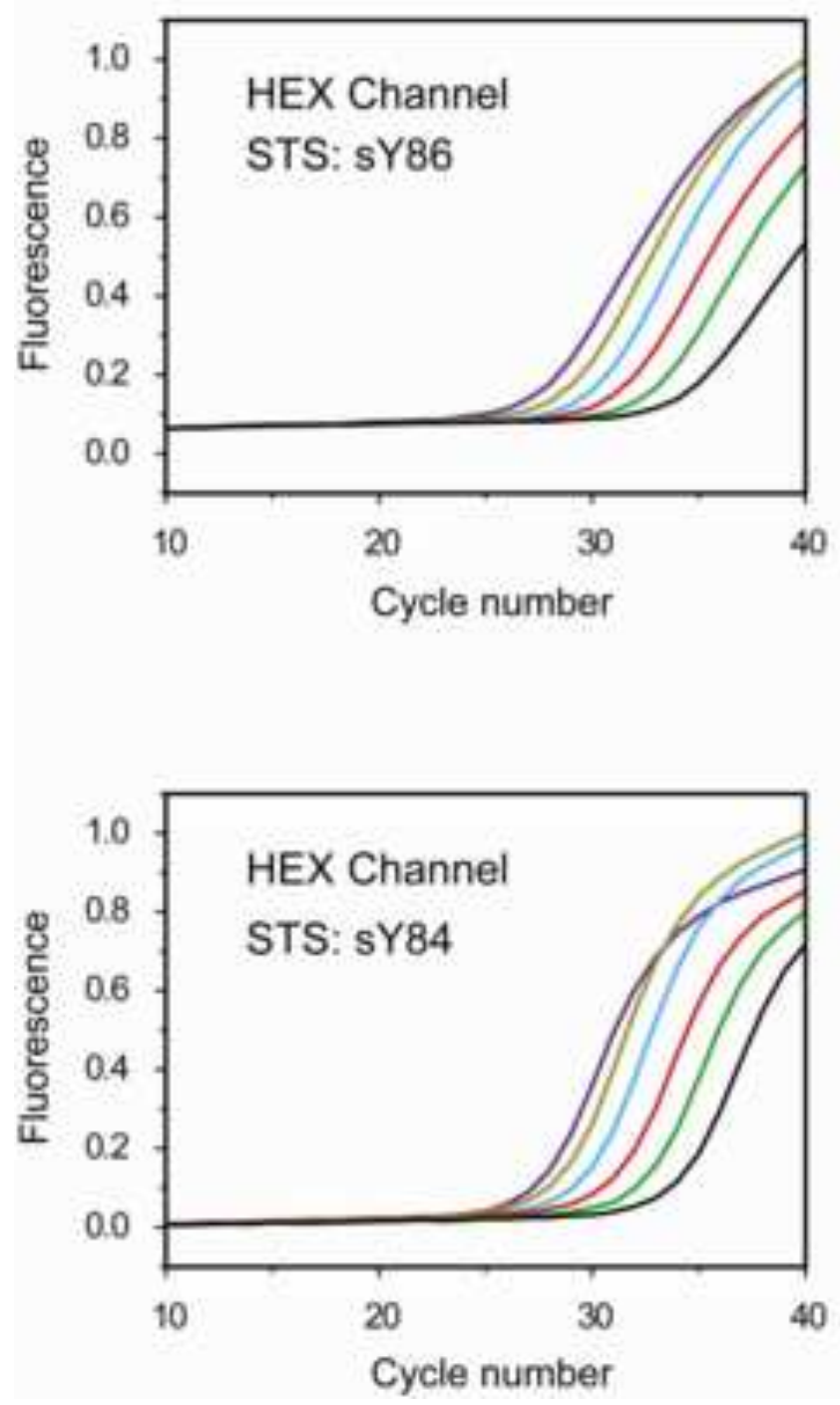

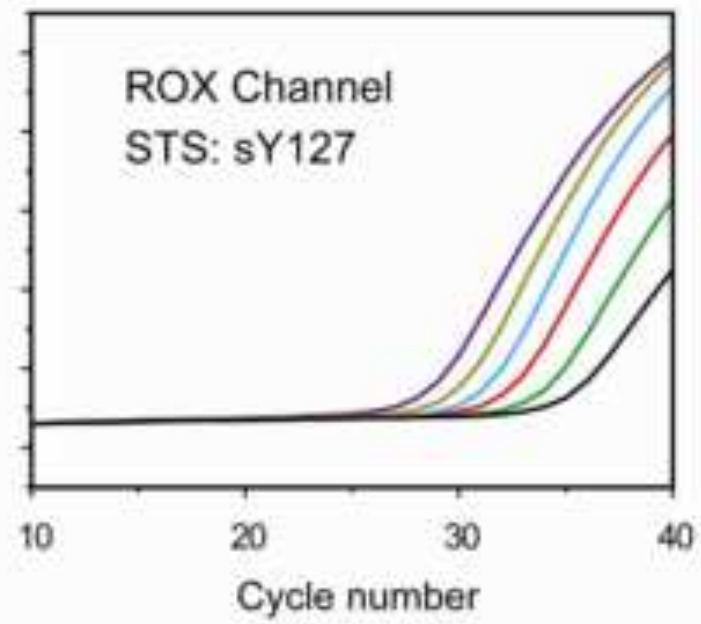

Reaction B

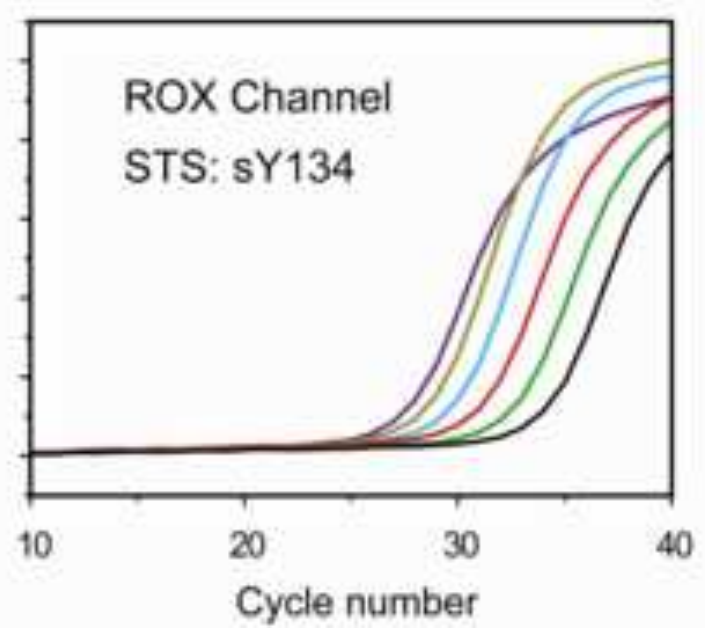

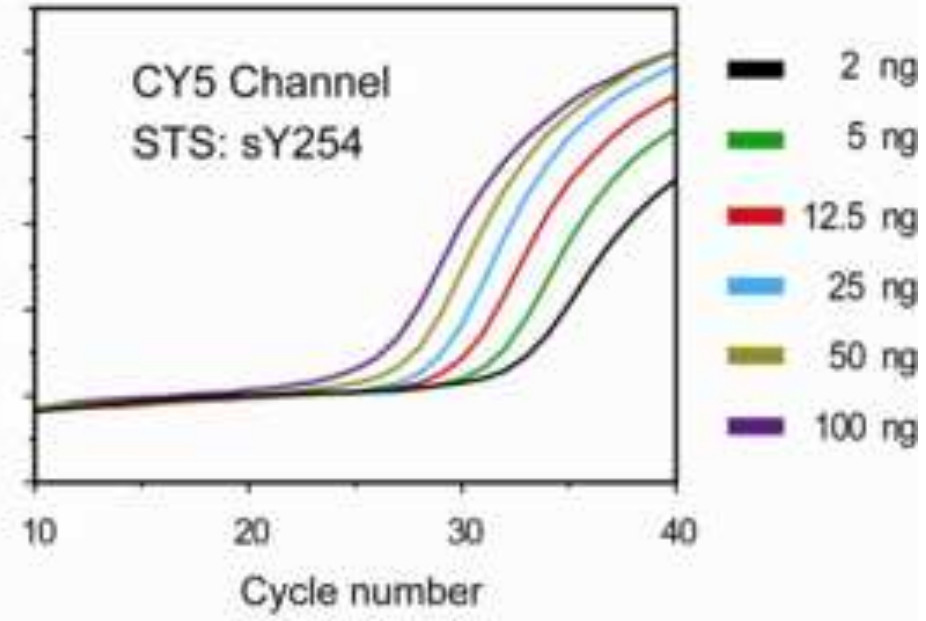

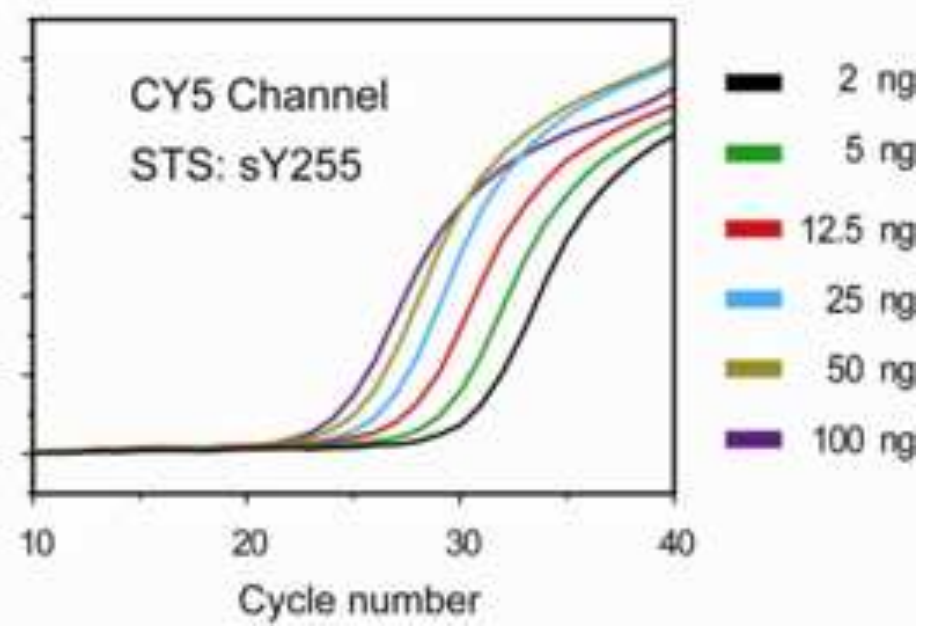


Reaction A
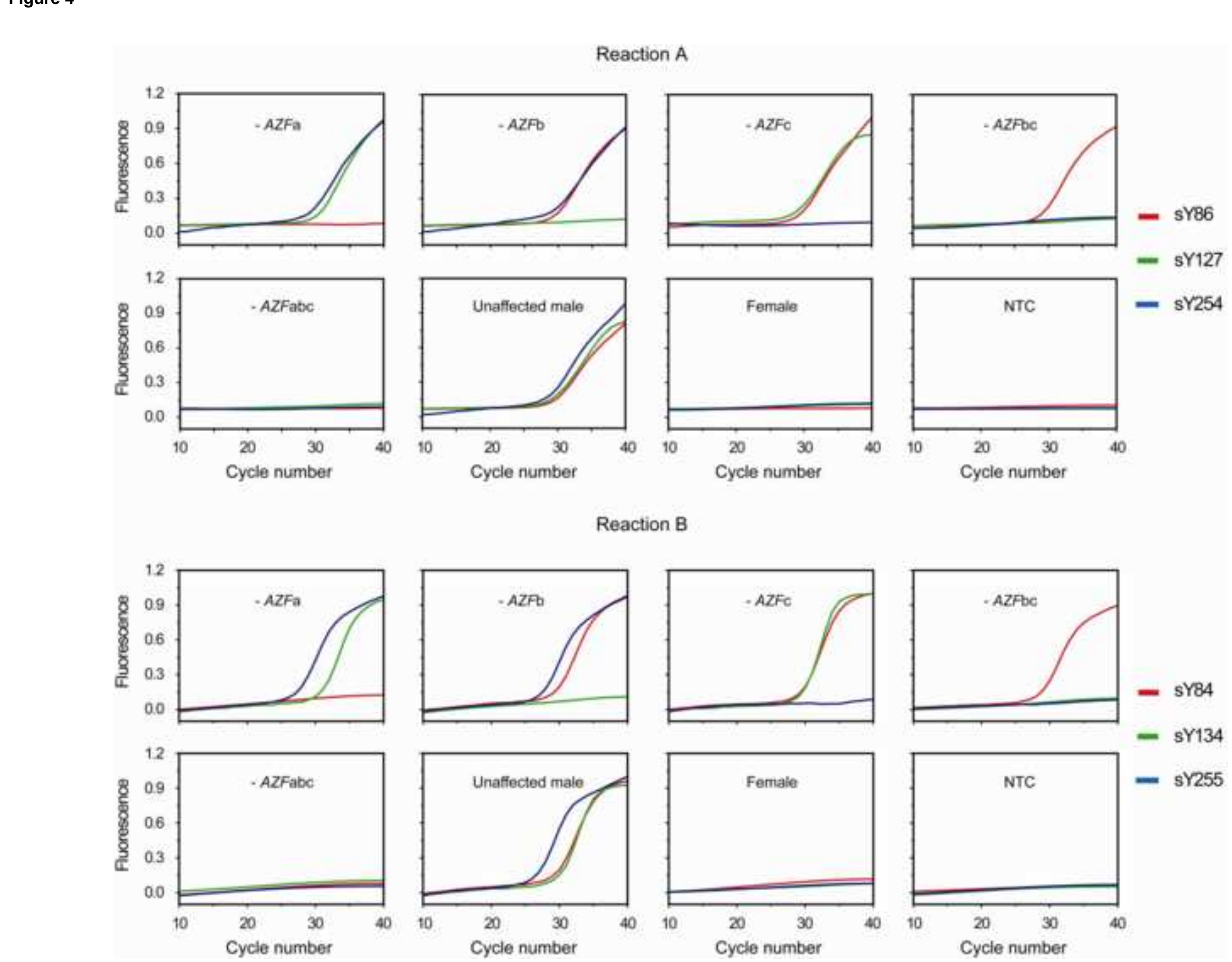

Cycle number
Reaction B

Cycle number
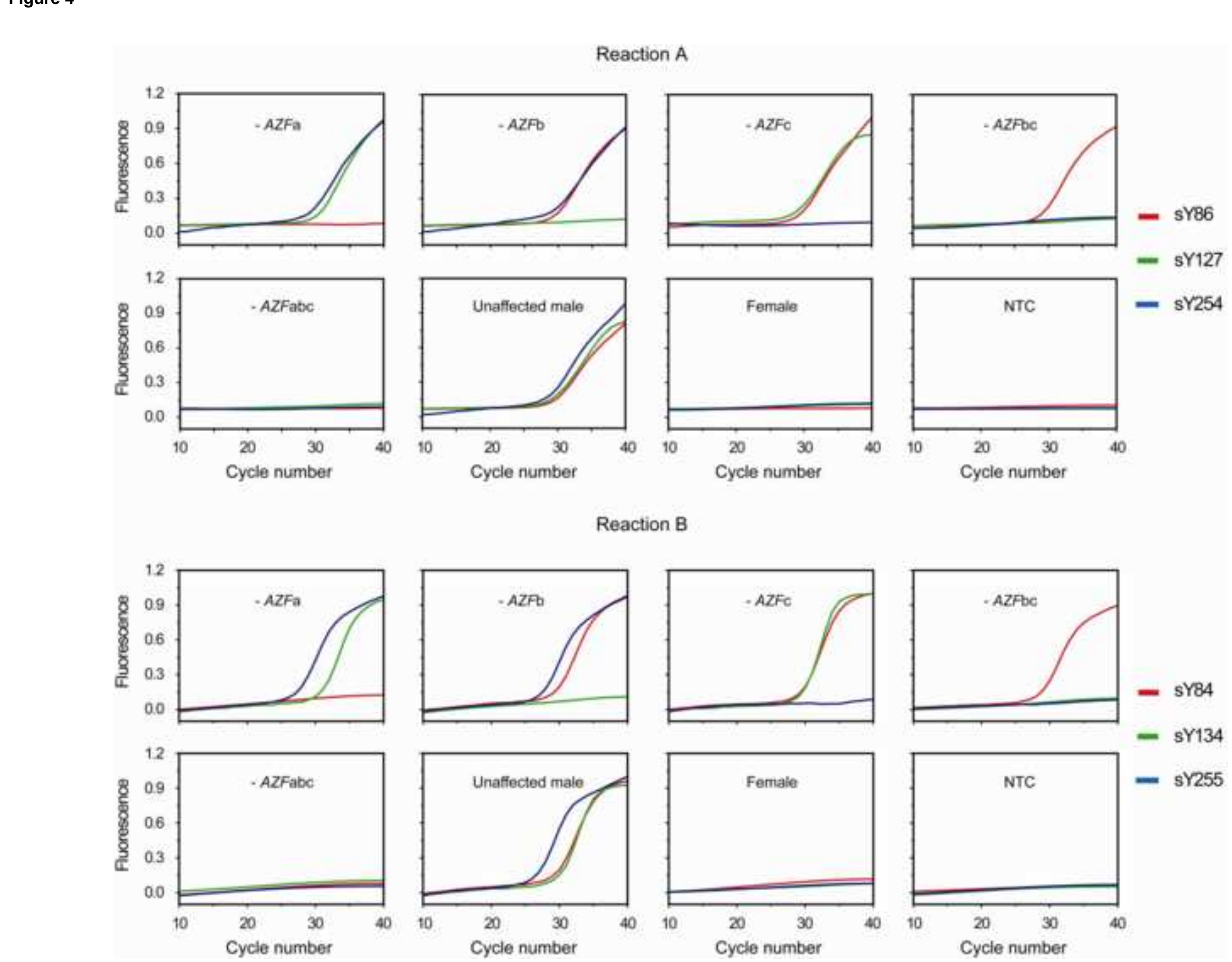

- sY84
- sY134

- sY255
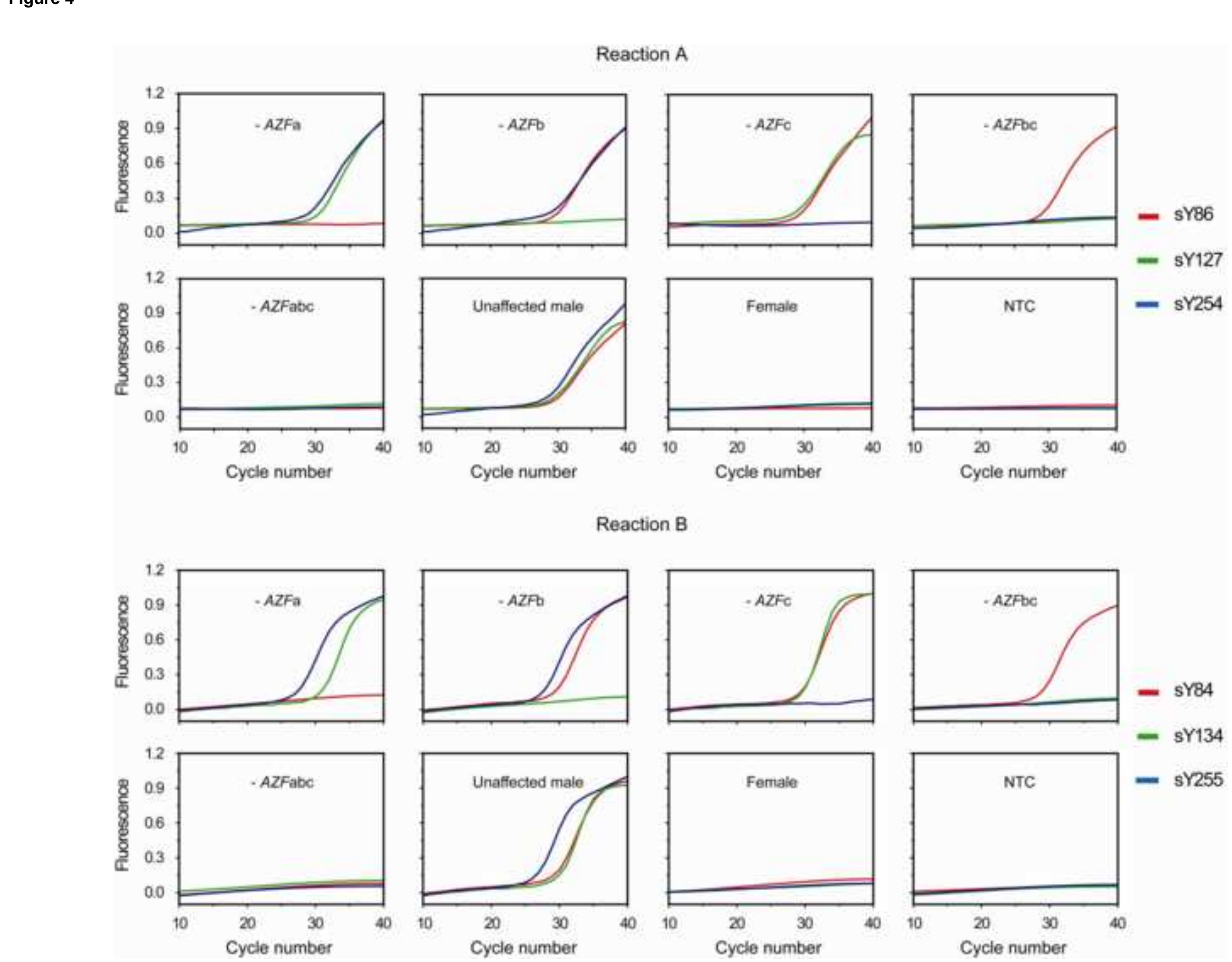

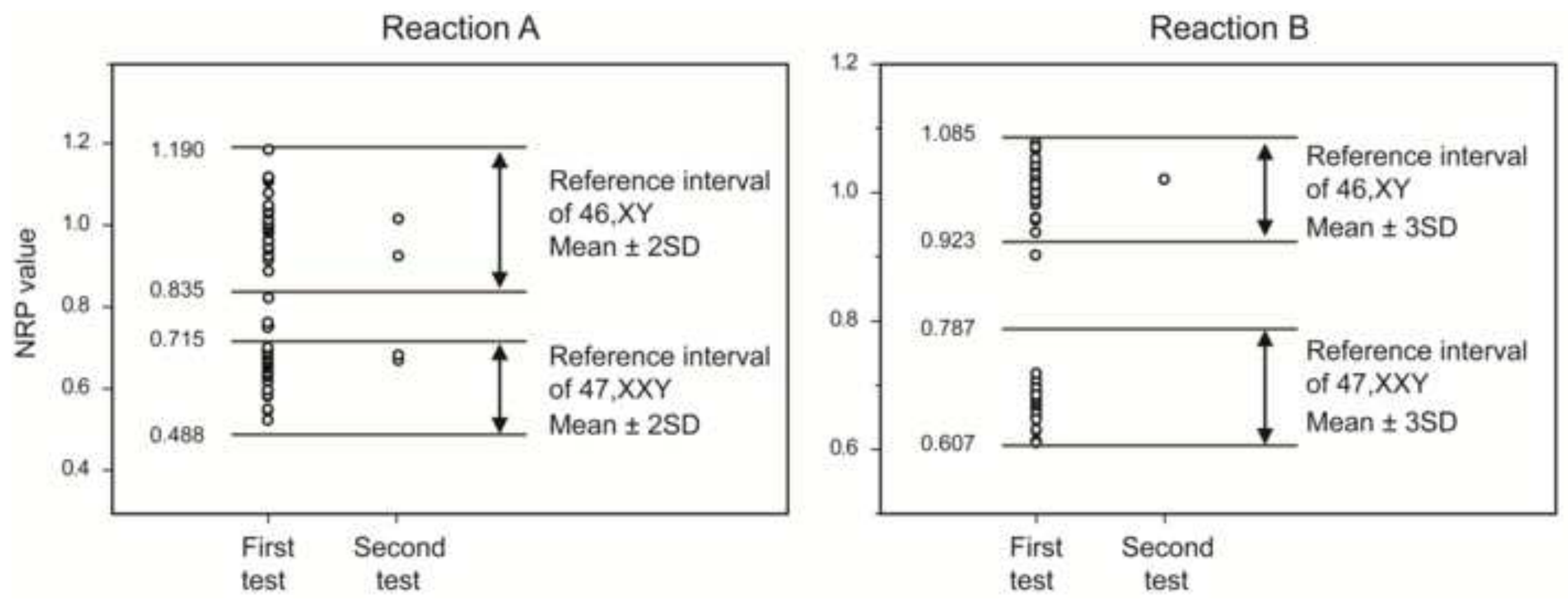
Table 1 - Primers and probes used in the Real-time quadruplex assay

\begin{tabular}{|c|c|c|}
\hline Name & Sequence ( 5 ' to 3 ') & $\begin{array}{c}\text { Concentration } \\
(\mathrm{nmol} / \mathrm{L})\end{array}$ \\
\hline \multicolumn{3}{|l|}{ Reaction A } \\
\hline $\mathrm{X} / \mathrm{Y}-1-\mathrm{F}$ & GCAAGCCCTCACGTAGCGAAGAACACCTTGCCAAGAAGAA & 80 \\
\hline $\mathrm{X} / \mathrm{Y}-1-\mathrm{R}$ & GCAAGCCCTCACGTAGCGAACAGCTTGTGGCTCTCCA & 800 \\
\hline $\mathrm{X} / \mathrm{Y}-1-\mathrm{P}$ & FAM-TACCGCTGTACTGAC-BHQ1 & 400 \\
\hline sY86-F & GCAAGCCCTCACGTAGCGAACTCACAGTCCTTGAGGCTA & 40 \\
\hline sY86-R & GCAAGCCCTCACGTAGCGAAAAGACAGCATCTACAACCCA & 40 \\
\hline sY86-P & HEX-AAGCTATGGCCAGGGCTGG-BHQ1 & 200 \\
\hline sY127-F & GCAAGCCCTCACGTAGCGAAGGCTCACAAACGAAAAGAAA & 400 \\
\hline sY127-R & GCAAGCCCTCACGTAGCGAACTTTTGTATAATTAGCATCTCATGAA & 400 \\
\hline sY127-P & ROX-ACTGGAATCTACCAAAGCCCACTGTGTTCATG-BHQ2 & 200 \\
\hline sY254-F & GCAAGCCCTCACGTAGCGAAGGGTGTTACCAGAAGGCAA & 40 \\
\hline sY254-R & GCAAGCCCTCACGTAGCGAACCCATTGTTCATGATGTATGTT & 40 \\
\hline sY254-P & CY5-TCGTGCCAAACACTGTTTTTGTTGGTGGAA-BHQ2 & 200 \\
\hline \multicolumn{3}{|l|}{ Reaction B } \\
\hline $\mathrm{X} / \mathrm{Y}-2-\mathrm{F}$ & GCAAGCCCTCACGTAGCGAAGCTAACACAGTGAAATCACCA & 80 \\
\hline $\mathrm{X} / \mathrm{Y}-2-\mathrm{R}$ & GCAAGCCCTCACGTAGCGAATGGCTGCATTTTGCAAT & 800 \\
\hline $\mathrm{X} / \mathrm{Y}-2-\mathrm{P}$ & FAM-TTCGTTCCCCTGAG-BHQ1 & 400 \\
\hline sY84-F & GCAAGCCCTCACGTAGCGAAGATTCAGTGGGACCCTTTCTT & 200 \\
\hline sY84-R & GCAAGCCCTCACGTAGCGAAGGAGGCTTCATCAGCAAGA & 200 \\
\hline sY84-P & HEX-AAGCTGGCTAACTCCTTTCAAAAGGTTTTGTCTT-BHQ1 & 200 \\
\hline sY134-F & GCAAGCCCTCACGTAGCGAAGAGGAATAGTACAGGTCAAAGGAA & 200 \\
\hline sY134-R & GCAAGCCCTCACGTAGCGAATCTTTCAGTCACAGAACGCTT & 200 \\
\hline sY134-P & $\begin{array}{l}\text { ROX-ATAGATGGGGTTGATACTAAAGTT- } \\
\text {-TAAAACATCTGGAACATTCTACT-BHQ2 }\end{array}$ & 200 \\
\hline sY255-F & GCAAGCCCTCACGTAGCGAAGTTACAGGATTCGGCGTGAT & 40 \\
\hline sY255-R & GCAAGCCCTCACGTAGCGAACTCGTCATGTGCAGCCAC & 40 \\
\hline sY255-P & CY5-AGGTAGGTTTCAGTGTTTGGATTCCGCA-BHQ2 & 200 \\
\hline Universal primer & GCAAGCCCTCACGTAGCGAA & 1600 \\
\hline
\end{tabular}

F: forward primer; R: reverse primer; P: probe. 
Table 2. Comparison of the results obtained with the quadruplex assay and reference methods for the detection of 47,XXY and $A Z F$ microdeletion samples

\begin{tabular}{|l|c|c|}
\hline DNA sample with: & $\begin{array}{c}\text { Number of samples detected } \\
\text { by quadruplex assay }\end{array}$ & $\begin{array}{c}\text { Number of samples detected } \\
\text { by reference methods } *\end{array}$ \\
\hline $47, \mathrm{XXY}$ & 30 & 30 \\
\hline$-A Z F$ a & 2 & 2 \\
\hline$-A Z F \mathrm{~b}$ & 1 & 1 \\
\hline$-A Z F \mathrm{c}$ & 14 & 7 \\
\hline$-A Z F$ bc & 7 & 5 \\
\hline$-A Z F$ abc & 5 & 30 \\
\hline $\begin{array}{l}46, \mathrm{XY} \text { without the } \\
\text { microdeletions }\end{array}$ & 30 & 14 \\
\hline
\end{tabular}

"karyotyping was used as a reference method for the detection of 47,XXY; EAA/EMQN guidelines method was used as a reference method for the detection of $A Z F$ microdeletions; please see the text of the MS for more details 